\title{
Dynamics of Almost Periodic BAM Neural Networks with Neutral Delays
}

\author{
Yaqin Li \\ Department of Mathematics, Kunming University, Kunming 650031, China \\ Correspondence should be addressed to Yaqin Li; yqlikm@163.com
}

Received 30 December 2013; Revised 6 April 2014; Accepted 6 April 2014; Published 28 April 2014

Academic Editor: Sabri Arik

Copyright (C) 2014 Yaqin Li. This is an open access article distributed under the Creative Commons Attribution License, which permits unrestricted use, distribution, and reproduction in any medium, provided the original work is properly cited.

The paper investigates the almost periodic oscillatory properties of neutral-type BAM neural networks with time-varying delays. By employing the contracting mapping principle and constructing suitable Lyapunov functional, several sufficient conditions are established for the existence, uniqueness, and global exponential stability of almost periodic solution of the system. The results of this paper are new and a simple example is given to illustrate the effectiveness of the new results.

\section{Introduction}

Recent years have witnessed rapid development of bidirectional associative memory (BAM) neural networks due to the vast applications in pattern recognition, artificial intelligence, automatic control engineering, and optimization because of its better abilities of information memory and information association [1-6]. It is well known that studies on neural dynamical systems not only involve a discussion of stability properties, but also involve many dynamic behaviors such as periodic oscillatory behavior, almost periodic oscillatory properties, chaos, and bifurcation. In applications, almost periodic oscillatory is more accordant with fact [7-9]. A great number of results for BAM neural networks concerning the existence and global stability of (almost) periodic solution have been derived (see, e.g., [10-16]).

In addition, owing to the complicated dynamic properties of the neural cells in the real world, the existing neural network models in many cases cannot characterize the properties of a neural reaction process precisely. It is natural and important that systems will contain some information about the derivative of the past state to further describe and model the dynamics for such complex neural reactions. This new type of neural networks is called neutral neural networks or neural networks of neutral type. The motivation for us to study neural networks of neutral type comes from three aspects. First, based on biochemistry experiments, neural information may transfer across chemical reactivity, which results in a neutral-type process. Second, in view of electronics, it has been shown that neutral phenomena exist in large-scale integrated (LSI) circuits. Last, the key point is that cerebra can be considered as a super LSI circuit with chemical reactivity, which reasonably implies that the neutral dynamic behaviors should be included in neural dynamic systems $[17,18]$. However, up to date, there are hardly any articles concerning the almost periodic oscillation analysis for neural networks of neutral type.

Motivated by the above reason, in this paper, we consider the following neutral-type BAM neural networks with timevarying delays:

$$
\begin{aligned}
\dot{x}_{i}(t)= & -a_{i}(t) x_{i}(t)+\sum_{j=1}^{m} w_{1 j i}(t) f_{j}\left(y_{j}\left(t-\alpha_{j}(t)\right)\right) \\
& +\sum_{j=1}^{n} w_{2 i j}(t) h_{j}\left(\dot{x}_{j}\left(t-\mu_{j}(t)\right)\right)+I_{i}(t), \\
\dot{y}_{j}(t)= & -b_{j}(t) y_{j}(t)+\sum_{i=1}^{n} v_{1 i j}(t) g_{i}\left(x_{i}\left(t-\beta_{i}(t)\right)\right) \\
& +\sum_{i=1}^{m} v_{2 j i}(t) k_{i}\left(\dot{y}_{i}\left(t-v_{i}(t)\right)\right)+J_{i}(t),
\end{aligned}
$$


where $a_{i}$ and $b_{j}$ denote the rate with which the cells $i$ and $j$ reset their potential to the resting states when isolated from the other cells and inputs, $w_{1 j i}, w_{2 i j^{\prime}}, v_{1 i j}$, and $v_{2 j i^{\prime}}$ are the connection weights at the time $t, I_{i}$ and $J_{j}$ denote the constant external inputs, $\alpha_{j} \geq 0$ and $\beta_{i} \geq 0$ are time delays in the state, $\mu_{i} \geq 0$ and $v_{j} \geq 0$ are neutral delays, $f_{j}, g_{i}, h_{i}$, and $k_{j}$ are the activation functions, and $a_{i}, b_{j}, w_{1 j i}, w_{2 i j}, v_{1 i j}, v_{2 j i}, f_{j}, g_{i}, h_{i}$, $k_{j}, \alpha_{j}, \beta_{i}, \mu_{i}, v_{j}, I_{i}$, and $J_{j}$ are all almost periodic functions, $i, j=1,2, \ldots, n, j, i=1,2, \ldots, m$.

Recently, there are many papers concerning the existence and exponential stability of (almost) periodic solution for BAM neural networks [10-16]. However, the research on the neutral-type BAM neural networks is few. Therefore, the main purpose of this paper is to establish some new sufficient conditions on the existence, uniqueness, and exponential stability of almost periodic solution of neutral-type BAM neural networks (1). First, by using the exponential dichotomy and the contracting mapping principle, the existence and uniqueness of almost periodic solution of system (1) is considered. Besides, by constructing a new Lyapunov functional, the stability criterion with system (1) is introduced. The methods used in this paper provide a possible method to study the existence and exponential stability of almost periodic solutions of neutral-type neural networks.

Let $C(\mathbb{X}, \mathbb{Y})$ and $C^{1}(\mathbb{X}, \mathbb{Y})$ be the space of continuous functions and continuously differential functions which map $\mathbb{X}$ into $\mathbb{Y}$, respectively. In particular, $C(\mathbb{X}):=C(\mathbb{X}, \mathbb{X})$ and $C^{1}(\mathbb{X}):=C^{1}(\mathbb{X}, \mathbb{X})$. For any bounded function $f \in C(\mathbb{R})$, $f^{u}=\sup _{s \in \mathbb{R}}|f(s)|$ and $f^{l}=\inf _{s \in \mathbb{R}}|f(s)|$.

Let $\sigma:=\max _{1 \leq i \leq n, 1 \leq j \leq m}\left\{\alpha_{j}^{u}, \beta_{i}^{u}, \mu_{i}^{u}, \nu_{j}^{u}\right\}$. The initial conditions associated with system (1) are of the form

$$
\begin{aligned}
& x_{i}(s)=\varphi_{i}^{*}(s), \quad \dot{x}_{i}(s)=\dot{\varphi}_{i}^{*}(s), \\
& \forall s \in[-\sigma, 0], \quad \varphi_{i}^{*} \in C^{1}([-\sigma, 0], \mathbb{R}), \quad i=1,2, \ldots, n, \\
& y_{j}(s)=\phi_{j}^{*}(s), \quad \dot{y}_{j}(s)=\dot{\phi}_{j}^{*}(s), \\
& \forall s \in[-\sigma, 0], \quad \phi_{j}^{*} \in C^{1}([-\sigma, 0], \mathbb{R}), \quad j=1,2, \ldots, m .
\end{aligned}
$$

Now we list some assumptions which will be used in this paper.

$\left(\mathrm{H}_{1}\right) \min _{1 \leq i \leq n, 1 \leq j \leq m}\left\{a_{i}^{l}, b_{j}^{l}\right\}>0$.

$\left(\mathrm{H}_{2}\right)$ There exist some positive constants $L_{j}^{f}, L_{i}^{g}, L_{i}^{h}$, and $L_{j}^{k}$ such that

$$
\begin{array}{r}
\left|f_{j}(u)-f_{j}(v)\right| \leq L_{j}^{f}|u-v|, \\
\left|k_{j}(u)-k_{j}(v)\right| \leq L_{j}^{k}|u-v|, \\
\left|g_{i}(u)-g_{i}(v)\right| \leq L_{i}^{g}|u-v|, \\
\left|h_{i}(u)-h_{i}(v)\right| \leq L_{i}^{h}|u-v|, \\
\forall u, v \in \mathbb{R},
\end{array}
$$

$\left(\mathrm{H}_{3}\right) \theta=\max \left\{\theta_{1}, \theta_{2}\right\}<1$, where

$$
\begin{aligned}
& \theta_{1}=\max _{1 \leq i \leq n, 1 \leq j \leq m}\left\{\sum_{j=1}^{m}\left(a_{i}^{l}\right)^{-1} w_{1 j i}^{u} L_{j}^{f}+\sum_{j=1}^{n}\left(a_{i}^{l}\right)^{-1} w_{2 i j}^{u} L_{j}^{h},\right.\left.\sum_{i=1}^{n}\left(b_{j}^{l}\right)^{-1} v_{1 i j}^{u} L_{i}^{g}+\sum_{i=1}^{m}\left(b_{j}^{l}\right)^{-1} v_{2 j i}^{u} L_{i}^{k}\right\}, \\
& \theta_{2}=\max _{1 \leq i \leq n, 1 \leq j \leq m}\left\{\left[1+\frac{a_{i}^{u}}{a_{i}^{l}}\right] \sum_{j=1}^{m}\left[w_{1 j i}^{u} L_{j}^{f}+\sum_{j=1}^{n} w_{2 i j}^{u} L_{j}^{h}\right],\right. \\
& {\left.\left[1+\frac{b_{j}^{u}}{b_{j}^{l}}\right]\left[\sum_{i=1}^{n} v_{1 i j}^{u} L_{i}^{g}+\sum_{i=1}^{m} v_{2 j i}^{u} L_{i}^{k}\right]\right\} . }
\end{aligned}
$$

By the basic theory of neutral functional differential equations in [17], the initial value problems (1) and (2) have a unique solution on interval $[\sigma,+\infty)$.

The organization of this paper is as follows. In Section 2, we give some basic definitions and necessary lemmas which will be used in later sections. In Sections 3 and 4, by using a fixed point theorem and constructing suitable Lyapunov functional, we obtain some sufficient conditions ensuring existence, uniqueness, and global exponential stability of almost periodic solution of system (1). Finally, an example is given to illustrate that our results are feasible.

\section{Preliminaries}

Now, let us state the following definitions and lemmas, which will be useful in proving our main result.

Definition 1 (see, [7]). $x \in C(\mathbb{R})$ is called almost periodic, if, for any $\epsilon>0$, it is possible to find a real number $l=l(\epsilon)>0$ and, for any interval with length $l(\epsilon)$, there exists a number $\tau=\tau(\epsilon)$ in this interval such that $|x(t+\tau)-x(t)|<\epsilon$, for all $t \in \mathbb{R}$. The collection of those functions is denoted by $A P(\mathbb{R})$.

Definition 2 (see, [7]). Let $y \in C\left(\mathbb{R}, \mathbb{R}^{n}\right)$ and $P(t)$ be a $n \times n$ continuous matrix defined on $\mathbb{R}$. The linear system

$$
\dot{y}(t)=P(t) y(t)
$$

is said to be an exponential dichotomy on $\mathbb{R}$ if there exist constants $k, \lambda>0$, projection $S$, and the fundamental matrix $Y(t)$ satisfying

$$
\begin{gathered}
\left\|Y(t) S Y^{-1}(s)\right\| \leq k e^{-\lambda(t-s)}, \quad \forall t \geq s, \\
\left\|Y(t)(I-S) Y^{-1}(s)\right\| \leq k e^{-\lambda(s-t)}, \quad \forall t \leq s .
\end{gathered}
$$

Lemma 3 (see, [7]). If the linear system $\dot{y}(t)=P(t) y(t)$ has an exponential dichotomy, then almost periodic system

$$
\dot{y}(t)=P(t) y(t)+g(t)
$$


has a unique almost periodic solution $y(t)$ which can be expressed as follows:

$$
\begin{aligned}
y(t)= & \int_{-\infty}^{t} Y(t) S Y^{-1}(s) g(s) \mathrm{d} s \\
& -\int_{t}^{\infty} Y(t)(I-S) Y^{-1}(s) g(s) \mathrm{d} s .
\end{aligned}
$$

Lemma 4 (see, [7]). Let $a_{i}(t)$ be an almost periodic function and

$$
M\left[a_{i}\right]=\lim _{T \rightarrow \infty} \frac{1}{T} \int_{t}^{t+T} a_{i}(s) \mathrm{d} s>0, \quad i=1,2, \ldots, n .
$$

Then the linear system $\dot{y}(t)=-A(t) y(t)$ admits an exponential dichotomy, where $A(t)=\operatorname{diag}\left\{a_{1}(t), a_{2}(t), \ldots, a_{n}(t)\right\}$.

Definition 5. The almost periodic solution $z=$ $\left(x_{1}, \ldots, x_{n}, y_{1}, \ldots, y_{m}\right)^{T}$ of system (1) with the initial value $z^{*}=\left(x_{1}^{*}, \ldots, x_{n}^{*}, y_{1}^{*}, \ldots, y_{m}^{*}\right)^{T}$ is said to be globally exponentially stable, if there exist constants $\omega>0$ and $M \geq 1$, for any solution $\phi=\left(\varphi_{1}, \ldots, \varphi_{n}, \psi_{1}, \ldots, \psi_{m}\right)^{T}$ of system (1) with initial value $\phi^{*}=\left(\varphi_{1}^{*}, \ldots, \varphi_{n}^{*}, \psi_{1}^{*}, \ldots, \psi_{m}^{*}\right)^{T}$ such that

$$
\sum_{i=1}^{n}\left|x_{i}(t)-\varphi_{i}(t)\right|+\sum_{j=1}^{m}\left|y_{j}(t)-\psi_{j}(t)\right| \leq M\left\|z^{*}-\phi^{*}\right\|_{\sigma} e^{-\omega t}
$$

where

$$
\begin{aligned}
&\left\|z^{*}-\phi^{*}\right\|_{\sigma} \\
&:=\max _{1 \leq i \leq n, 1 \leq j \leq m_{s \in[-\sigma, 0]}} \sup _{s}\left\{\left|x_{i}^{*}(s)-\varphi_{i}^{*}(s)\right|+\left|\dot{x}_{i}^{*}(s)-\dot{\varphi}_{i}^{*}(s)\right|,\right. \\
&\left.\left|y_{j}^{*}(s)-\psi_{j}^{*}(s)\right|+\left|\dot{y}_{j}^{*}(s)-\dot{\psi}_{j}^{*}(s)\right|\right\} .
\end{aligned}
$$

Lemma 6 (see, [19]). Assume that $\mathbb{X}$ is a Banach space with norm $\|\cdot\| ; T: \mathbb{X} \rightarrow \mathbb{X}$ is a contraction mapping; that is, there exists $k \in(0,1)$, such that

$$
\|T x-T y\| \leq k\|x-y\|, \quad \forall x, y \in \mathbb{X} .
$$

Then $T$ has a unique fixed point in $\mathbb{X}$.

Let $\mathbb{E}=\left\{x \in A P(\mathbb{R}) \cap C^{1}(\mathbb{R}): \dot{x} \in A P(\mathbb{R})\right\}$ and

$$
\begin{aligned}
\mathbb{X}=\{z & =\left(x_{1}, \ldots, x_{n}, y_{1}, \ldots, y_{m}\right)^{T} \\
& \left.: x_{i}, y_{j} \in \mathbb{E}, i=1, \ldots, n, j=1, \ldots, m\right\},
\end{aligned}
$$

with the norm

$$
\begin{aligned}
\|z\|=\max \left\{\|z\|_{0},\|\dot{z}\|_{0}\right\} & \\
=\max \left\{\max _{1 \leq i \leq n, 1 \leq j \leq m}\left\{\left|x_{i}\right|_{0},\left|y_{j}\right|_{0}\right\},\right. & \left.\max _{1 \leq i \leq n, 1 \leq j \leq m}\left\{\left|\dot{x}_{i}\right|_{0},\left|\dot{y}_{j}\right|_{0}\right\}\right\},
\end{aligned}
$$

where $\|z\|_{0}=\max _{1 \leq i \leq n, 1 \leq j \leq m}\left\{\left|x_{i}\right|_{0},\left|y_{j}\right|_{0}\right\},\|\dot{z}\|_{0}=$ $\max _{1 \leq i \leq n, 1 \leq j \leq m}\left\{\left|\dot{x}_{i}\right|_{0},\left|\dot{y}_{j}\right|_{0}\right\}$, and $|f|_{0}=\sup _{s \in \mathbb{R}}|f(s)|$, for all $f \in A P(\mathbb{R})$. Then $\mathbb{X}$ is a Banach space with the norm $\|\cdot\|$.

By Lemmas 3 and 4, system (1) has a unique almost periodic solution

$$
z^{\phi}=\left(x_{1}^{\varphi_{1}}, \ldots, x_{n}^{\varphi_{n}}, y_{1}^{\psi_{1}}, \ldots, y_{m}^{\psi_{n}}\right)^{T}
$$

which can be expressed as follows:

$$
\begin{aligned}
x_{i}^{\varphi_{i}} & =\int_{-\infty}^{t} e^{-\int_{s}^{t} a_{i}(u) \mathrm{d} u} F_{i}(s, \phi(s)) \mathrm{d} s, \\
y_{j}^{\psi_{j}} & =\int_{-\infty}^{t} e^{-\int_{s}^{t} b_{j}(u) \mathrm{d} u} G_{j}(s, \phi(s)) \mathrm{d} s,
\end{aligned}
$$

where $\phi=\left(\varphi_{1}, \ldots, \varphi_{n}, \psi_{1}, \ldots, \psi_{m}\right)^{T}$,

$$
\begin{aligned}
F_{i}(t, \phi(t))= & \sum_{j=1}^{m} w_{1 j i}(t) f_{j}\left(\psi_{j}\left(t-\alpha_{j}(t)\right)\right) \\
& +\sum_{j=1}^{n} w_{2 i j}(t) h_{j}\left(\dot{\varphi}_{j}\left(t-\mu_{j}(t)\right)\right)+I_{i}(t),
\end{aligned}
$$$$
G_{j}(t, \phi(t))=\sum_{i=1}^{n} v_{1 i j}(t) g_{i}\left(\varphi_{i}\left(t-\beta_{i}(t)\right)\right)
$$

$$
+\sum_{i=1}^{m} v_{2 j i}(t) k_{i}\left(\dot{\psi}_{i}\left(t-v_{i}(t)\right)\right)+J_{i}(t)
$$

where $i=1,2, \ldots, n, j=1,2, \ldots, m$, and $t \in \mathbb{R}$.

Let $T: \mathbb{X} \rightarrow \mathbb{X}$ be defined by

$$
\begin{aligned}
T(\phi)= & \left(\Phi_{1}(\phi), \ldots, \Phi_{n}(\phi), \Psi_{1}(\phi), \ldots, \Psi_{m}(\phi)\right)^{T} \\
= & \left(x_{1}^{\varphi_{1}}, \ldots, x_{n}^{\varphi_{n}}, y_{1}^{\psi_{1}}, \ldots, y_{m}^{\psi_{n}}\right)^{T}, \\
& \forall \phi=\left(\varphi_{1}, \ldots, \varphi_{n}, \psi_{1}, \ldots, \psi_{m}\right)^{T} \in \mathbb{X} .
\end{aligned}
$$

\section{Existence and Uniqueness}

In this section, we study the existence and uniqueness of almost periodic solution of system (1).

Theorem 7. Assume that $\left(H_{1}\right)-\left(H_{3}\right)$ hold, then system (1) has a unique almost periodic solution.

Proof. Consider the following nonlinear operator:

$$
\begin{array}{r}
T(\phi)=\left(\Phi_{1}(\phi), \ldots, \Phi_{n}(\phi), \Psi_{1}(\phi), \ldots, \Psi_{m}(\phi)\right)^{T}, \\
\forall \phi=\left(\varphi_{1}, \ldots, \varphi_{n}, \psi_{1}, \ldots, \psi_{m}\right)^{T} \in \mathbb{X} .
\end{array}
$$


For all $\phi^{p}=\left(\varphi_{1}^{p}, \ldots, \varphi_{n}^{p}, \psi_{1}^{p}, \ldots, \psi_{m}^{p}\right)^{T} \in \mathbb{X}, p=1,2$, it follows from the definitions of $F_{i}$ and $G_{j}$ that

$$
\begin{aligned}
& \left|F_{i}\left(t, \phi^{1}\right)-F_{i}\left(t, \phi^{2}\right)\right|_{0} \\
& \quad=\sup _{s \in \mathbb{R}}\left|F_{i}\left(s, \phi^{1}\right)-F_{i}\left(s, \phi^{2}\right)\right| \\
& \quad \leq \sum_{j=1}^{m} w_{1 j i}^{u} L_{j}^{f}\left|\psi_{j}^{1}-\psi_{j}^{2}\right|_{0}+\sum_{j=1}^{n} w_{2 i j}^{u} L_{j}^{h}\left|\dot{\varphi}_{j}^{1}-\dot{\varphi}_{j}^{2}\right|_{0}, \\
& \left|G_{j}\left(t, \phi^{1}\right)-G_{j}\left(t, \phi^{2}\right)\right|_{0} \\
& \quad=\sup _{s \in \mathbb{R}}\left|G_{j}\left(s, \phi^{1}\right)-G_{j}\left(s, \phi^{2}\right)\right| \\
& \quad \leq \sum_{i=1}^{n} v_{1 i j}^{u} L_{i}^{g}\left|\varphi_{i}^{1}-\varphi_{i}^{2}\right|_{0}+\sum_{i=1}^{m} v_{2 j i}^{u} L_{i}^{k}\left|\dot{\psi}_{i}^{1}-\dot{\psi}_{i}^{2}\right|_{0} .
\end{aligned}
$$

Then

$$
\begin{aligned}
& \left|\Phi_{i}\left(\phi^{1}\right)-\Phi_{i}\left(\phi^{2}\right)\right|_{0} \\
& =\sup _{t \in \mathbb{R}}\left|\Phi_{i}\left(\phi^{1}(t)\right)-\Phi_{i}\left(\phi^{1}(t)\right)\right| \\
& \leq \sup _{t \in \mathbb{R}} \mid \int_{-\infty}^{t} e^{-\int_{s}^{t} a_{i}(u) \mathrm{d} u} \\
& \quad \times\left[F_{i}\left(s, \phi^{1}(s)\right)-F_{i}\left(s, \phi^{2}(s)\right)\right] \mathrm{d} s \mid \\
& \leq \sum_{j=1}^{m}\left(a_{i}^{l}\right)^{-1} w_{1 j i}^{u} L_{j}^{f}\left|\psi_{j}^{1}-\psi_{j}^{2}\right|_{0} \\
& \quad+\sum_{j=1}^{n}\left(a_{i}^{l}\right)^{-1} w_{2 i j}^{u} L_{j}^{h}\left|\dot{\varphi}_{j}^{1}-\dot{\varphi}_{j}^{2}\right|_{0}, \\
& \left|\Psi_{j}\left(\phi^{1}\right)-\Psi_{j}\left(\phi^{2}\right)\right|_{0} \\
& =\sup _{t \in \mathbb{R}}\left|\Psi_{j}\left(\phi^{1}(t)\right)-\Psi_{j}\left(\phi^{2}(t)\right)\right| \\
& \leq \sup _{t \in \mathbb{R}} \mid \int_{-\infty}^{t} e^{-\int_{s}^{t} b_{j}(u) \mathrm{d} u} \\
& \quad \times\left[G_{j}\left(s, \phi^{1}(s)\right)-G_{j}\left(s, \phi^{2}(s)\right)\right] \mathrm{d} s \mid \\
& \leq \sum_{i=1}^{n}\left(b_{j}^{l}\right)^{-1} v_{1 i j}^{u} L_{i}^{g}\left|\varphi_{i}^{1}-\varphi_{i}^{2}\right|_{0} \\
& \quad \sum_{j}^{l}\left(b_{j}^{l}\right)^{-1} v_{2 j i}^{u} L_{i}^{k}\left|\dot{\psi}_{i}^{1}-\dot{\psi}_{i}^{2}\right|_{0},
\end{aligned}
$$

where $i=1,2, \ldots, n$ and $j=1,2, \ldots, m$.
Further, we also obtain that

$$
\begin{aligned}
& \left|\dot{\Phi}_{i}\left(\phi^{1}\right)-\dot{\Phi}_{i}\left(\phi^{2}\right)\right|_{0} \\
& =\sup _{t \in \mathbb{R}}\left|\dot{\Phi}_{i}\left(\phi^{1}(t)\right)-\dot{\Phi}_{i}\left(\phi^{2}(t)\right)\right| \\
& =\sup _{t \in \mathbb{R}} \mid-a_{i}(t)\left[\Phi_{i}\left(\phi^{1}\right)-\Phi_{i}\left(\phi^{2}\right)\right] \\
& \quad+\left[F_{i}\left(t, \phi^{1}\right)-F_{i}\left(t, \phi^{2}\right)\right] \mid \\
& \leq\left[1+\frac{a_{i}^{u}}{a_{i}^{l}}\right]\left[\sum_{j=1}^{m} w_{1 j i}^{u} L_{j}^{f}\left|\psi_{j}^{1}-\psi_{j}^{2}\right|_{0}\right. \\
& \left.\quad+\sum_{j=1}^{n} w_{2 i j}^{u} L_{j}^{h}\left|\dot{\varphi}_{j}^{1}-\dot{\varphi}_{j}^{2}\right|_{0}\right] \\
& \leq\left[1+\frac{a_{i}^{u}}{a_{i}^{l}}\right]\left[\sum_{j=1}^{m} w_{1 j i}^{u} L_{j}^{f}+\sum_{j=1}^{n} w_{2 i j}^{u} L_{j}^{h}\right]\left\|\phi^{1}-\phi^{2}\right\|,
\end{aligned}
$$

$\left|\dot{\Psi}_{j}\left(\phi^{1}\right)-\dot{\Psi}_{j}\left(\phi^{2}\right)\right|_{0}$

$$
=\sup _{t \in \mathbb{R}}\left|\dot{\Psi}_{j}\left(\phi^{1}(t)\right)-\dot{\Psi}_{j}\left(\phi^{2}(t)\right)\right|
$$$$
=\sup _{t \in \mathbb{R}} \mid-b_{j}(t)\left[\Psi_{j}\left(\phi^{1}\right)-\Psi_{j}\left(\phi^{2}\right)\right]
$$$$
+\left[G_{j}\left(t, \phi^{1}\right)-G_{j}\left(t, \phi^{2}\right)\right] \mid
$$$$
\leq\left[1+\frac{b_{j}^{u}}{b_{j}^{l}}\right]\left[\sum_{i=1}^{n} v_{1 i j}^{u} L_{i}^{g}\left|\varphi_{i}^{1}-\varphi_{i}^{2}\right|_{0}\right.
$$$$
\left.+\sum_{i=1}^{m} v_{2 j i}^{u} L_{i}^{k}\left|\dot{\psi}_{i}^{1}-\dot{\psi}_{i}^{2}\right|_{0}\right]
$$$$
\leq\left[1+\frac{b_{j}^{u}}{b_{j}^{l}}\right]\left[\sum_{i=1}^{n} v_{1 i j}^{u} L_{i}^{g}+\sum_{i=1}^{m} v_{2 j i}^{u} L_{i}^{k}\right]\left\|\phi^{1}-\phi^{2}\right\|,
$$

where $i=1, \ldots, n$ and $j=1, \ldots, m$.

$$
\text { Hence, }
$$

$$
\begin{aligned}
& \left\|T\left(\phi^{1}\right)-T\left(\phi^{2}\right)\right\|_{0} \\
& =\max _{1 \leq i \leq n, 1 \leq j \leq m}\left\{\left|\Phi_{i}\left(\phi^{1}\right)-\Phi_{i}\left(\phi^{2}\right)\right|_{0}, \Psi_{j}\left(\phi^{1}\right)-\left.\Psi_{j}\left(\phi^{2}\right)\right|_{0}\right\} \\
& \leq \max _{1 \leq i \leq n, 1 \leq j \leq m}\left\{\sum_{j=1}^{m}\left(a_{i}^{l}\right)^{-1} w_{1 j i}^{u} L_{j}^{f}+\sum_{j=1}^{n}\left(a_{i}^{l}\right)^{-1} w_{2 i j}^{u} L_{j}^{h},\right. \\
& \left.\quad \sum_{i=1}^{n}\left(b_{j}^{l}\right)^{-1} v_{1 i j}^{u} L_{i}^{g}+\sum_{i=1}^{m}\left(b_{j}^{l}\right)^{-1} v_{2 j i}^{u} L_{i}^{k}\right\} \\
& \quad \times\left\|\phi^{1}-\phi^{2}\right\| \\
& =\theta_{1}\left\|\phi^{1}-\phi^{2}\right\|,
\end{aligned}
$$




$$
\begin{aligned}
& \left\|\dot{T}\left(\phi^{1}\right)-\dot{T}\left(\phi^{2}\right)\right\|_{0} \\
& =\max _{1 \leq i \leq n, 1 \leq j \leq m}\left\{\left|\dot{\Phi}_{i}\left(\phi^{1}\right)-\dot{\Phi}_{i}\left(\phi^{2}\right)\right|_{0}, \dot{\Psi}_{j}\left(\phi^{1}\right)-\left.\dot{\Psi}_{j}\left(\phi^{2}\right)\right|_{0}\right\} \\
& \leq \max _{1 \leq i \leq n, 1 \leq j \leq m}\left\{\left[1+\frac{a_{i}^{u}}{a_{i}^{l}}\right] \sum_{j=1}^{m}\left[w_{1 j i}^{u} L_{j}^{f}+\sum_{j=1}^{n} w_{2 i j}^{u} L_{j}^{h}\right],\right. \\
& \left.\quad\left[1+\frac{b_{j}^{u}}{b_{j}^{l}}\right]\left[\sum_{i=1}^{n} v_{1 i j}^{u} L_{i}^{g}+\sum_{i=1}^{m} v_{2 j i}^{u} L_{i}^{k}\right]\right\} \\
& \quad \times\left\|\phi^{1}-\phi^{2}\right\| \\
& =\theta_{2}\left\|\phi^{1}-\phi^{2}\right\| .
\end{aligned}
$$

Together with the above results, one has

$$
\begin{aligned}
& \left\|T\left(\phi^{1}\right)-T\left(\phi^{2}\right)\right\| \\
& \quad=\max \left\{\left\|T\left(\phi^{1}\right)-T\left(\phi^{2}\right)\right\|_{0},\left\|\dot{T}\left(\phi^{1}\right)-\dot{T}\left(\phi^{2}\right)\right\|_{0}\right\} \\
& \quad \leq \max \left\{\theta_{1}, \theta_{2}\right\}\left\|\phi^{1}-\phi^{2}\right\| \\
& \quad \leq \theta\left\|\phi^{1}-\phi^{2}\right\|,
\end{aligned}
$$

where $\theta \in(0,1)$. By Lemma 6, there exists a unique fixed point $\phi_{0} \in \mathbb{X}$ satisfying $T\left(\phi_{0}\right)=\phi_{0}$, which implies that system (1) has unique almost periodic solution. This completes the proof.

Remark 8. Condition $\left(\mathrm{H}_{3}\right)$ in Theorem 7 indicates that the neutral terms are harmful for the existence and uniqueness of almost periodic solution of system (1).

\section{Global Exponential Stability}

Theorem 9. Assume that $\left(H_{1}\right)-\left(H_{3}\right)$ hold and suppose further the following.

$\left(\mathrm{H}_{4}\right) \alpha_{j}, \beta_{i}, \mu_{i}$, and $v_{j}$ are differential functions, with $\dot{\alpha}_{j}^{+}:=$ $\sup _{s \in \mathbb{R}} \dot{\alpha}_{j}(s)<1, \dot{\beta}_{i}^{+}:=\sup _{s \in \mathbb{R}} \dot{\beta}_{i}(s)<1, \dot{\mu}_{i}^{+}:=$ $\sup _{s \in \mathbb{R}} \dot{\mu}_{i}(s)<1, \dot{v}_{j}^{+}:=\sup _{s \in \mathbb{R}} \dot{\nu}_{j}(s)<1$, where $i=$ $1,2, \ldots, n$ and $j=1,2, \ldots, m$.

$\left(\mathrm{H}_{5}\right)$ There exists a positive constant $\lambda \leq 1$ such that

$$
\begin{aligned}
& -(1-\lambda) a_{i}^{l}+(1+\lambda) \sum_{j=1}^{m} \frac{v_{1 i j}^{u} L_{i}^{g}}{1-\dot{\beta}_{i}^{+}}<0, \quad i=1,2, \ldots, n, \\
& -(1-\lambda) b_{j}^{l}+(1+\lambda) \sum_{i=1}^{n} \frac{w_{1 j i}^{u} L_{j}^{f}}{1-\dot{\alpha}_{j}^{+}}<0, \quad j=1,2, \ldots, m,
\end{aligned}
$$$$
\sum_{i=1}^{n}(1+\lambda) w_{2 i j}^{u} L_{j}^{h}-\lambda\left(1-\dot{\mu}_{j}^{+}\right)<0, \quad j=1,2, \ldots, m,
$$

$$
\sum_{j=1}^{m}(1+\lambda) v_{2 j i}^{u} L_{i}^{k}-\lambda\left(1-\dot{v}_{i}^{+}\right)<0, \quad i=1,2, \ldots, n .
$$

Then system (1) has a unique almost periodic solution, which is globally exponentially stable.

Proof. It follows from Theorem 7 that system (1) has a unique almost periodic solution $\phi=\left(\varphi_{1}, \ldots, \varphi_{n}, \psi_{1}, \ldots, \psi_{m}\right)^{T}$ with initial value $\phi^{*}=\left(\varphi_{1}^{*}, \ldots, \varphi_{n}^{*}, \psi_{1}^{*}, \ldots, \psi_{m}^{*}\right)^{T}$. We next show that the almost periodic solution $\phi$ is globally exponentially stable.

Make a transformation for system (1): $x_{i}=u_{i}-\varphi_{i}$, $y_{j}=v_{j}-\psi_{j}, i=1,2, \ldots, n, j=1,2, \ldots, m$, where $z=\left(u_{1}, \ldots, u_{n}, v_{1}, \ldots, v_{m}\right)^{T}$ is arbitrary solution of system (1) with initial value $z^{*}=\left(u_{1}^{*}, \ldots, u_{n}^{*}, v_{1}^{*}, \ldots, v_{m}^{*}\right)^{T}$.

By $\left(\mathrm{H}_{4}\right)$, there exists a small enough positive constant $\omega$ such that

$$
\begin{gathered}
\omega-(1-\lambda) a_{i}^{l}+(1+\lambda) \sum_{j=1}^{m} \frac{v_{1 i j}^{u} L_{i}^{g} e^{\omega \beta_{i}^{u}}}{1-\dot{\beta}_{i}^{+}}<0, \quad i=1,2, \ldots, n, \\
\omega-(1-\lambda) b_{j}^{l}+(1+\lambda) \sum_{i=1}^{n} \frac{w_{1 j i}^{u} L_{j}^{f} e^{\omega \alpha_{j}^{u}}}{1-\dot{\alpha}_{j}^{+}}<0, \quad j=1,2, \ldots, m, \\
\sum_{i=1}^{n}(1+\lambda) w_{2 i j}^{u} L_{j}^{h}-\lambda e^{-\omega \mu_{j}^{u}}\left(1-\dot{\mu}_{j}^{+}\right)<0, \quad j=1,2, \ldots, m, \\
\sum_{j=1}^{m}(1+\lambda) v_{2 j i}^{u} L_{i}^{k}-\lambda e^{-\omega v_{i}^{u}}\left(1-\dot{v}_{i}^{+}\right)<0, \quad i=1,2, \ldots, n .
\end{gathered}
$$

Define

$$
V_{1}(t)=\sum_{i=1}^{n} e^{\omega t}\left|x_{i}(t)\right|+\sum_{j=1}^{m} e^{\omega t}\left|y_{j}(t)\right|
$$

In view of system (1), we have

$$
\begin{aligned}
& D^{+} V_{1}(t) \\
& \leq \omega \sum_{i=1}^{n} e^{\omega t}\left|x_{i}(t)\right|+\omega \sum_{j=1}^{m} e^{\omega t}\left|y_{j}(t)\right| \\
& +\sum_{i=1}^{n} e^{\omega t}\left[-a_{i}^{l}\left|x_{i}(t)\right|+\sum_{j=1}^{m} w_{1 j i}^{u} L_{j}^{f}\left|y_{j}\left(t-\alpha_{j}(t)\right)\right|\right. \\
& \left.+\sum_{j=1}^{n} w_{2 i j}^{u} L_{j}^{h}\left|\dot{x}_{j}\left(t-\mu_{j}(t)\right)\right|\right] \\
& +\sum_{j=1}^{m} e^{\omega t}\left[-b_{j}^{l}\left|y_{j}(t)\right|+\sum_{i=1}^{n} v_{1 i j}^{u} L_{i}^{g}\left|x_{i}\left(t-\beta_{i}(t)\right)\right|\right. \\
& \left.+\sum_{i=1}^{m} v_{2 j i}^{u} L_{i}^{k}\left|\dot{y}_{i}\left(t-v_{i}(t)\right)\right|\right] .
\end{aligned}
$$




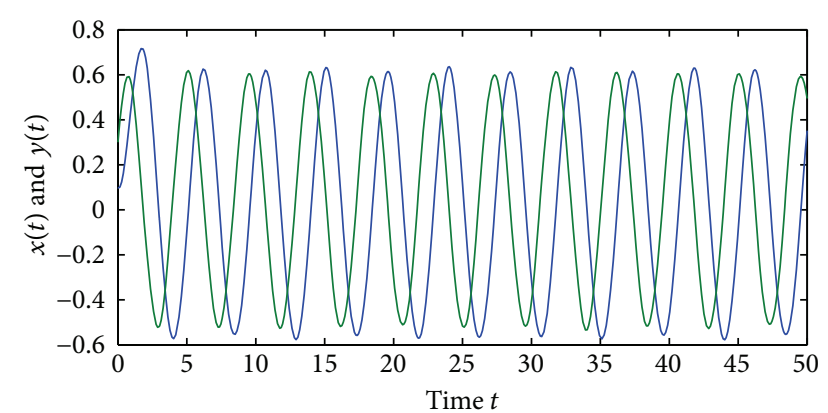

FIGURE 1: Almost periodicity of state variables $x(t)$ and $y(t)$.

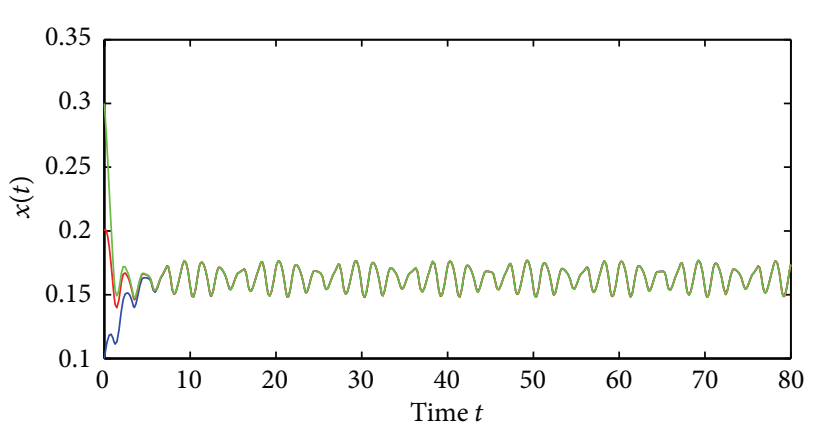

FIGURE 2: Stability of state variables $x(t)$.

Let

$V_{2}(t)=\lambda \sum_{i=1}^{n} \int_{t-\mu_{i}(t)}^{t} e^{\omega s}\left|\dot{x}_{i}(s)\right| \mathrm{d} s$

$V_{3}(t)=\lambda \sum_{j=1}^{m} \int_{t-v_{j}(t)}^{t} e^{\omega s}\left|\dot{y}_{j}(s)\right| \mathrm{d} s$

$V_{4}(t)=\sum_{j=1}^{m} \sum_{i=1}^{n}(1+\lambda) \int_{t-\beta_{i}(t)}^{t} \frac{v_{1 i j}^{u} L_{i}^{g}}{1-\dot{\beta}_{i}^{+}} e^{\omega\left(s+\beta_{i}^{u}\right)}\left|x_{i}(s)\right| \mathrm{d} s$

$V_{5}(t)=\sum_{i=1}^{n} \sum_{j=1}^{m}(1+\lambda) \int_{t-\alpha_{j}(t)}^{t} \frac{w_{1 j i}^{u} L_{j}^{f}}{1-\dot{\alpha}_{j}^{+}} e^{\omega\left(s+\alpha_{j}^{u}\right)}\left|y_{j}(s)\right| \mathrm{d} s$.

So

$$
\begin{aligned}
& D^{+} V_{2}(t) \\
& \leq \lambda \sum_{i=1}^{n} e^{\omega t}\left|\dot{x}_{i}(t)\right| \\
& \quad-\lambda \sum_{i=1}^{n} e^{\omega\left(t-\mu_{i}(t)\right)}\left(1-\dot{\mu}_{i}(t)\right)\left|\dot{x}_{i}\left(t-\mu_{i}(t)\right)\right|
\end{aligned}
$$

$$
\begin{aligned}
& \leq \lambda \sum_{i=1}^{n} e^{\omega t}\left[a_{i}^{u}\left|x_{i}(t)\right|+\sum_{j=1}^{m} w_{1 j i}^{u} L_{j}^{f}\left|y_{j}\left(t-\alpha_{j}(t)\right)\right|\right] \\
& \quad-\lambda \sum_{j=1}^{n} e^{\omega t}\left[e^{-\omega \mu_{j}^{u}}\left(1-\dot{\mu}_{j}^{+}\right)-\sum_{i=1}^{n} w_{2 i j}^{u} L_{j}^{h}\right]\left|\dot{x}_{j}\left(t-\mu_{j}(t)\right)\right|,
\end{aligned}
$$

$D^{+} V_{4}(t)$

$$
\begin{aligned}
& \leq \sum_{j=1}^{m} \sum_{i=1}^{n}(1+\lambda) \frac{v_{1 i j}^{u} L_{i}^{g}}{1-\dot{\beta}_{i}^{+}} e^{\omega\left(t+\beta_{i}^{u}\right)}\left|x_{i}(t)\right| \\
& \quad-\sum_{j=1}^{m} \sum_{i=1}^{n}(1+\lambda) \frac{v_{1 i j}^{u} L_{i}^{g}\left(1-\dot{\beta}_{i}(t)\right)}{1-\dot{\beta}_{i}^{+}} e^{\omega\left(t-\beta_{i}(t)+\beta_{i}^{u}\right)} \\
& \quad \times\left|x_{i}\left(t-\beta_{i}(t)\right)\right| \\
& \leq \sum_{j=1}^{m} \sum_{i=1}^{n}(1+\lambda) \frac{v_{1 i j}^{u} L_{i}^{g}}{1-\dot{\beta}_{i}^{+}} e^{\omega\left(t+\beta_{i}^{u}\right)}\left|x_{i}(t)\right| \\
& \quad-\sum_{j=1}^{m} \sum_{i=1}^{n}(1+\lambda) v_{1 i j}^{u} L_{i}^{g} e^{\omega t}\left|x_{i}\left(t-\beta_{i}(t)\right)\right| .
\end{aligned}
$$

Similar to the arguments as that in (30) and (31), we obtain

$$
\begin{aligned}
& D^{+} V_{3}(t) \\
& \leq \lambda \sum_{j=1}^{m} e^{\omega t}\left[b_{j}^{u}\left|y_{j}(t)\right|+\sum_{i=1}^{n} v_{1 i j}^{u} L_{i}^{g}\left|x_{i}\left(t-\beta_{i}(t)\right)\right|\right] \\
& \quad-\lambda \sum_{i=1}^{m} e^{\omega t}\left[e^{-\omega v_{i}^{u}}\left(1-\dot{v}_{i}^{+}\right)-\sum_{j=1}^{m} v_{2 j i}^{u} L_{i}^{k}\right]\left|\dot{y}_{i}\left(t-v_{i}(t)\right)\right|,
\end{aligned}
$$

$D^{+} V_{5}(t)$

$$
\begin{aligned}
\leq & \sum_{i=1}^{n} \sum_{j=1}^{m}(1+\lambda) \frac{w_{1 j i}^{u} L_{j}^{f}}{1-\dot{\alpha}_{j}^{+}} e^{\omega\left(t+\alpha_{j}^{u}\right)}\left|y_{j}(t)\right| \\
& -\sum_{i=1}^{n} \sum_{j=1}^{m}(1+\lambda) w_{1 j i}^{u} L_{j}^{f} e^{\omega t}\left|y_{j}\left(t-\alpha_{j}(t)\right)\right| .
\end{aligned}
$$

Define $V(t)=\sum_{q=1}^{5} V_{q}$. From (28)-(33), it follows that $D^{+} V(t)$

$$
\begin{aligned}
& \leq e^{\omega t} \sum_{i=1}^{n}\left[\omega-(1-\lambda) a_{i}^{l}+(1+\lambda) \sum_{j=1}^{m} \frac{v_{1 i j}^{u} L_{i}^{g} e^{\omega \beta_{i}^{u}}}{1-\beta_{i}^{\prime+}}\right]\left|x_{i}(t)\right| \\
& +e^{\omega t} \sum_{j=1}^{m}\left[\omega-(1-\lambda) b_{j}^{l}+(1+\lambda) \sum_{i=1}^{n} \frac{w_{1 j i}^{u} L_{j}^{f} e^{\omega \alpha_{j}^{u}}}{1-\dot{\alpha}_{j}^{+}}\right]
\end{aligned}
$$




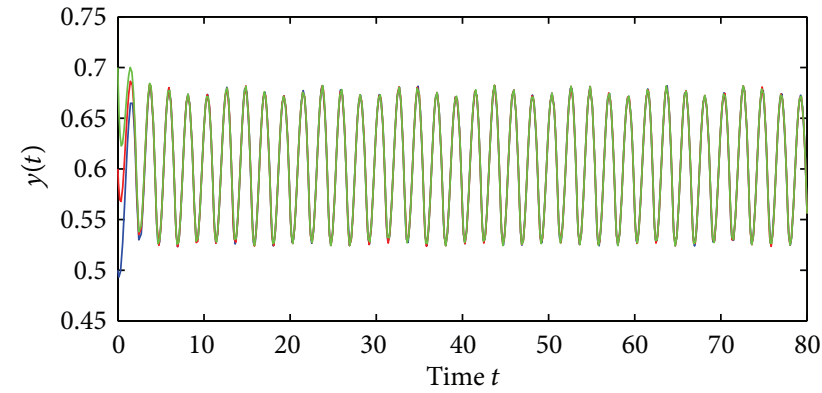

FIGURE 3: Stability of state variables $y(t)$.

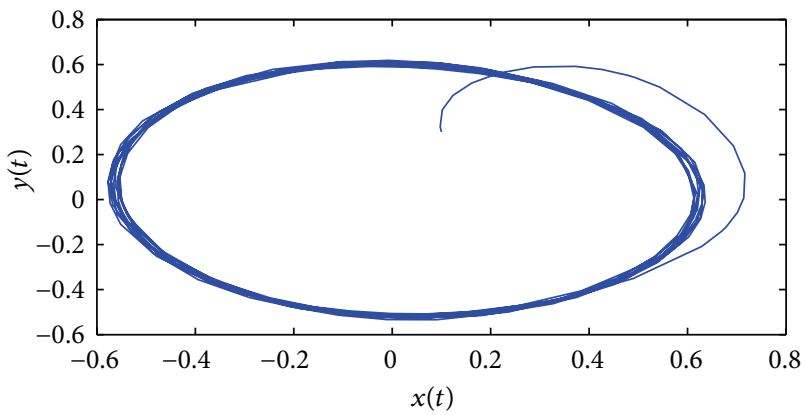

FIGURE 4: Phase response of state variables $x(t)$ and $y(t)$.

$$
\begin{aligned}
& \times\left|y_{j}(t)\right| \\
& +e^{\omega t} \sum_{j=1}^{n}\left[\sum_{i=1}^{n}(1+\lambda) w_{2 i j}^{u} L_{j}^{h}-\lambda e^{-\omega \mu_{j}^{u}}\left(1-\dot{\mu}_{j}^{+}\right)\right] \\
& \times\left|\dot{x}_{j}\left(t-\mu_{j}(t)\right)\right| \\
& +e^{\omega t} \sum_{i=1}^{m}\left[\sum_{j=1}^{m}(1+\lambda) v_{2 j i}^{u} L_{i}^{k}-\lambda e^{-\omega v_{i}^{u}}\left(1-\dot{v}_{i}^{+}\right)\right] \\
& \times\left|\dot{y}_{i}\left(t-v_{i}(t)\right)\right| \\
& \leq 0,
\end{aligned}
$$

which implies that $V(t) \leq V(0)$, for all $t>0$. Obviously,

$$
\sum_{i=1}^{n} e^{\omega t}\left|x_{i}(t)\right|+\sum_{j=1}^{m} e^{\omega t}\left|y_{j}(t)\right| \leq V(t)
$$

On the other hand, we have

$V(0)$

$$
\begin{aligned}
= & \sum_{i=1}^{n}\left|x_{i}(0)\right|+\sum_{j=1}^{m}\left|y_{j}(0)\right|+\lambda \sum_{i=1}^{n} \int_{-\mu_{i}(0)}^{0}\left|\dot{x}_{i}(s)\right| \mathrm{d} s \\
& +\lambda \sum_{j=1}^{m} \int_{-v_{j}(0)}^{0}\left|\dot{y}_{j}(s)\right| \mathrm{d} s
\end{aligned}
$$

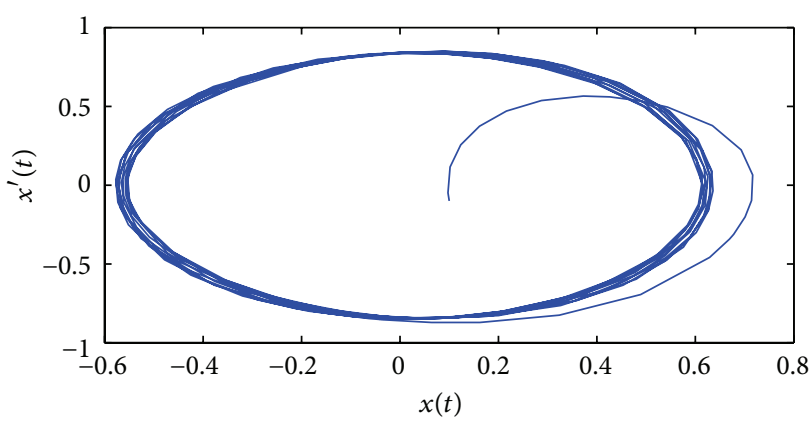

Figure 5: Phase response of state variables $x(t)$ and $x^{\prime}(t)$.

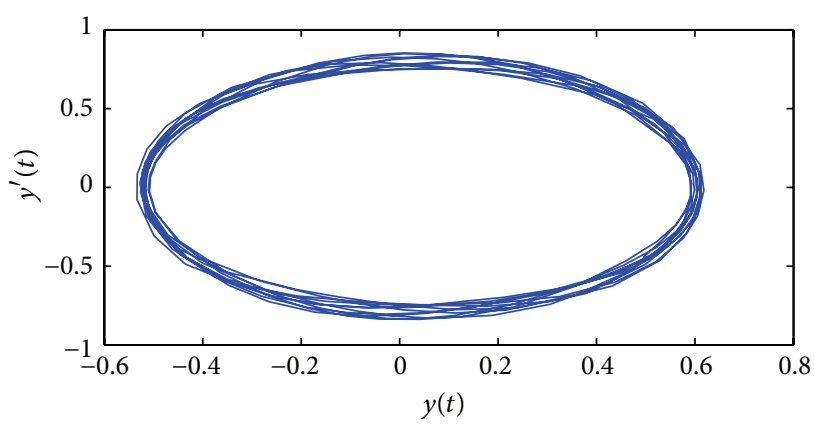

FIGURE 6: Phase response of state variables $y(t)$ and $y^{\prime}(t)$.

$$
\begin{aligned}
& +\sum_{j=1}^{m} \sum_{i=1}^{n}(1+\lambda) \int_{-\beta_{i}(0)}^{0} \frac{v_{1 i j}^{u} L_{i}^{g}}{1-\dot{\beta}_{i}^{+}} e^{\omega_{2}\left(s+\beta_{i}^{u}\right)}\left|x_{i}(s)\right| \mathrm{d} s \\
& +\sum_{i=1}^{n} \sum_{j=1}^{m}(1+\lambda) \int_{-\alpha_{j}(0)}^{0} \frac{w_{1 j i}^{u} L_{j}^{f}}{1-\dot{\alpha}_{j}^{+}} e^{\omega\left(s+\alpha_{j}^{u}\right)}\left|y_{j}(s)\right| \mathrm{d} s \\
& \leq\left\{m+n+\sum_{i=1}^{n} \mu_{i}^{u}+\sum_{j=1}^{m} v_{j}^{u}\right. \\
& \left.\quad+\sum_{j=1}^{m} \sum_{i=1}^{n}\left[\frac{2 \beta_{i}^{u} v_{1 i j}^{u} L_{i}^{g} e^{\omega \beta_{i}^{u}}}{1-\dot{\beta}_{i}^{+}}+\frac{2 \alpha_{j}^{u} w_{1 j i}^{u} L_{j}^{f} e^{\omega \alpha_{j}^{u}}}{1-\dot{\alpha}_{j}^{+}}\right]\right\} \\
& \times\left\|z^{*}-\phi^{*}\right\|,
\end{aligned}
$$

which implies from (35) that

$$
\sum_{i=1}^{n}\left|x_{i}(t)\right|+\sum_{j=1}^{m}\left|y_{j}(t)\right| \leq M\left\|z^{*}-\phi^{*}\right\| e^{-\omega t}, \quad \forall t>0,
$$

where

$$
\begin{aligned}
M:= & +n+\sum_{i=1}^{n} \mu_{i}^{u}+\sum_{j=1}^{m} \nu_{j}^{u} \\
& +\sum_{j=1}^{m} \sum_{i=1}^{n}\left[\frac{2 \beta_{i}^{u} v_{1 i j}^{u} L_{i}^{g} e^{\omega \beta_{i}^{u}}}{1-\dot{\beta}_{i}^{+}}+\frac{2 \alpha_{j}^{u} w_{1 j i}^{u} L_{j}^{f} e^{\omega \alpha_{j}^{u}}}{1-\dot{\alpha}_{j}^{+}}\right] .
\end{aligned}
$$




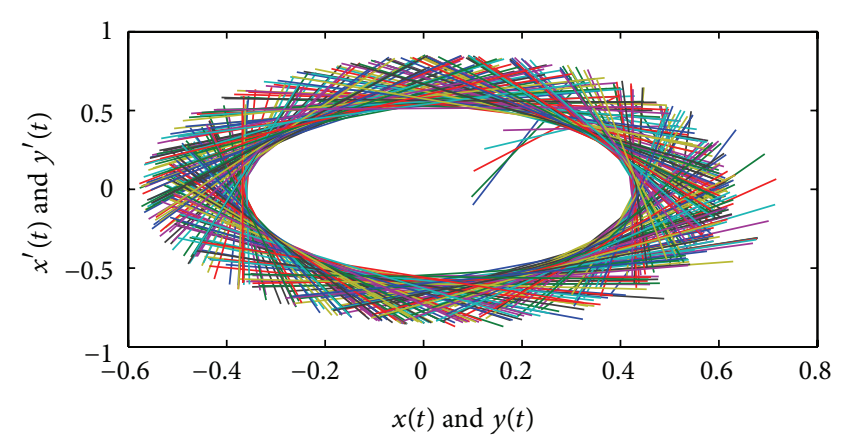

FIGURE 7: Phase response of state variables $x(t), y(t), x^{\prime}(t)$, and $y^{\prime}(t)$.

Thus, the almost periodic solution of system (1) is globally exponentially stable. This completes the proof.

Remark 10. Condition $\left(\mathrm{H}_{5}\right)$ in Theorem 9 indicates that the neutral terms and time delays are harmful for the global exponential stability of almost periodic solution of system (1).

\section{An Example}

Example 1. Consider the following neutral BAM neural networks with time-varying delays:

$$
\begin{aligned}
\dot{x}(t)= & -x(t)+\sin (\sqrt{2} t) f(y(t-\alpha(t))) \\
& +\cos ^{2}(\sqrt{3} t) h(\dot{x}(t-1))+\sin (\sqrt{2} t), \\
\dot{y}(t)= & -y(t)+|\cos (\sqrt{3} t)| g(x(t-\beta(t))) \\
& +\sin ^{2}(\sqrt{5} t) k(\dot{y}(t-1))+\cos (\sqrt{2} t),
\end{aligned}
$$

where $f(s)=g(s)=0.1 s$,

$$
\begin{aligned}
\left(\begin{array}{l}
h(s) \\
k(s)
\end{array}\right) & =\left(\begin{array}{l}
0.1 \sin (s) \\
0.1 \cos (s)
\end{array}\right), \\
\left(\begin{array}{l}
\alpha(s) \\
\beta(s)
\end{array}\right) & =\left(\begin{array}{l}
1+0.01 \sin ^{2}(\sqrt{2} s) \\
1+0.01 \cos ^{2}(\sqrt{3} s)
\end{array}\right),
\end{aligned}
$$

$\forall s \in \mathbb{R}$.

Then system (39) has a unique almost periodic solution, which is globally exponentially stable.

Proof. Corresponding to system (1), $a^{l}=b^{l}=1, L^{f}=L^{g}=$ $L^{h}=L^{k}=0.1, w_{1}^{u}=w_{2}^{u}=v_{1}^{u}=v_{2}^{u}=1, \dot{\alpha}^{+} \leq 0.02, \dot{\beta}^{+} \leq$ 0.02 , and $\dot{\mu}^{+}=\dot{\nu}^{+}=0$. Taking $\lambda=0.5$, it is easy to verify that $\left(\mathrm{H}_{1}\right)-\left(\mathrm{H}_{5}\right)$ hold and the results follow from Theorems 79 (see Figures 1, 2, 3, 4, 5, 6, and 7). This completes the proof.

For numerical simulation, Figures 1-3 depict the time responses of state variables of $x(t)$ and $y(t)$ with step $h=$ 0.01 of system (39), respectively. Figures 4-7 depict the phase responses of state variables $x(t), y(t), x^{\prime}(t)$, and $y^{\prime}(t)$, respectively. It confirms that the proposed conditions in our results are effective for system (39).

\section{Discussion}

In this paper, the neutral BAM neural network is considered. By employing fixed point theory and constructing suitable Lyapunov functional some new sufficient conditions are obtained for the existence and global exponential stability of almost periodic solution of the system. Conditions $\left(\mathrm{H}_{3}\right)$ and $\left(\mathrm{H}_{5}\right)$ in Theorems 7 and 9 indicate that the neutral terms and time delays are harm for the existence, uniqueness, and global exponential stability of almost periodic solution of the neutral-type system. The method used in this paper provides a possible method to study the existence and global exponential stability of almost periodic solution of other neutral neural networks (with impulses [20-23]).

\section{Conflict of Interests}

The author declares that there is no conflict of interests regarding the publication of this paper.

\section{Acknowledgments}

The author thanks the referee for his/her careful reading of the original paper and valuable comments and suggestions that greatly improved the presentation of this work. This work is supported by the Research Program of Kunming University in China (XJL13007).

\section{References}

[1] B. Kosko, "Adaptive bidirectional associative memories," Applied Optics, vol. 26, no. 23, pp. 4947-4960, 1987.

[2] B. Kosko, "Bidirectional associative memories," IEEE Transactions on Systems, Man, and Cybernetics, vol. 18, no. 1, pp. 49-60, 1988.

[3] X. F. Liao and K.-W. Wong, "Robust stability of interval bidirectional associative memory neural network with time delays," IEEE Transactions on Systems, Man, and Cybernetics, B: Cybernetics, vol. 34, no. 2, pp. 1142-1154, 2004.

[4] Q. K. Song and J. D. Cao, "Global exponential stability of bidirectional associative memory neural networks with distributed delays," Journal of Computational and Applied Mathematics, vol. 202, no. 2, pp. 266-279, 2007.

[5] H. Wang, Q. Song, and C. Duan, "LMI criteria on exponential stability of BAM neural networks with both time-varying delays and general activation functions," Mathematics and Computers in Simulation, vol. 81, no. 4, pp. 837-850, 2010.

[6] X. He, C. D. Li, T. W. Huang, C. J. Li, and J. J. Huang, "A recurrent neural network for solving bilevel linear programming problem," IEEE Transactions on Neural Networks and Learning Systems, vol. 25, pp. 824-830, 2014.

[7] A. M. Fink, Almost Periodic Differential Equations, vol. 377 of Lecture Notes in Mathematics, Springer, 1974.

[8] R. Ortega, "Degree theory and almost periodic problems," in Differential Equations, Chaos and Variational Problems, vol. 75 of Progress in Nonlinear Differential Equations and Their Applications, pp. 345-356, 2008. 
[9] X. Wang and H. Zhang, "A new approach to the existence, nonexistence and uniqueness of positive almost periodic solution for a model of hematopoiesis," Nonlinear Analysis. Real World Applications, vol. 11, no. 1, pp. 60-66, 2010.

[10] Y. K. Li, "Existence and exponential stability of periodic solution for continuous-time and discrete-time generalized bidirectional neural networks," Electronic Journal of Differential Equations, vol. 2006, pp. 1-21, 2006.

[11] W. G. Yang, "Existence and stability of periodic solutions of bam high-order hopfield neural networks with impulses and delays on time scales," Electronic Journal of Differential Equations, vol. 2012, pp. 1-22, 2012.

[12] A. P. Chen, L. H. Huang, and J. D. Cao, "Existence and stability of almost periodic solution for BAM neural networks with delays," Applied Mathematics and Computation, vol. 137, no. 1, pp. 177-193, 2003.

[13] Y. H. Xia, J. D. Cao, and M. R. Lin, "New results on the existence and uniqueness of almost periodic solution for BAM neural networks with continuously distributed delays," Chaos, Solitons \& Fractals, vol. 31, no. 4, pp. 928-936, 2007.

[14] L. J. Zhang and L. G. Si, "Existence and exponential stability of almost periodic solution for BAM neural networks with variable coefficients and delays," Applied Mathematics and Computation, vol. 194, no. 1, pp. 215-223, 2007.

[15] Y. K. Li and X. L. Fan, "Existence and globally exponential stability of almost periodic solution for Cohen-Grossberg BAM neural networks with variable coefficients," Applied Mathematical Modelling, vol. 33, no. 4, pp. 2114-2120, 2009.

[16] Y. K. Li, "Existence and stability of periodic solution for BAM neural networks with distributed delays," Applied Mathematics and Computation, vol. 159, no. 3, pp. 847-862, 2004.

[17] Z. M. Zhen, Functional Differential Equations, Anhui Education Press, Anhui, China, 1994, (Chinese).

[18] Y. Zhang, L. Guo, and C. Feng, "Stability analysis on a neutral neural network model," in Advances in Intelligent Computing, vol. 3644 of Lecture Notes in Computer Science, pp. 697-706, 2005.

[19] D. J. Guo, Nonlinear Functional Analysis, Shandong Science and Technology Press, Jinan, China, 2003, (Chinese).

[20] R. Sakthivel, R. Raja, and S. M. Anthoni, "Linear matrix inequality approach to stochastic stability of uncertain delayed BAM neural networks," IMA Journal of Applied Mathematics, vol. 78, no. 6, pp. 1156-1178, 2013.

[21] R. Sakthivel, R. Anbuvithya, K. Mathiyalagan, A. Arunkumar, and P. Prakash, "New LMI-based passivity criteria for neutraltype BAM neural networks with randomly occurring uncertainties," Reports on Mathematical Physics, vol. 72, no. 3, pp. 263286, 2013.

[22] R. Sakthivel, R. Raja, and S. M. Anthoni, "Exponential stability for delayed stochastic bidirectional associative memory neural networks with Markovian jumping and impulses," Journal of Optimization Theory and Applications, vol. 150, no. 1, pp. 166187, 2011.

[23] R. Sakthivel, R. Samidurai, and S. M. Anthoni, "New exponential stability criteria for stochastic BAM neural networks with impulses," Physica Scripta, vol. 82, no. 4, Article ID 045802, 2010. 


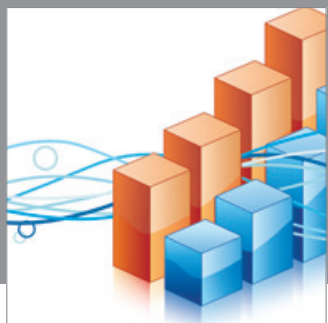

Advances in

Operations Research

mansans

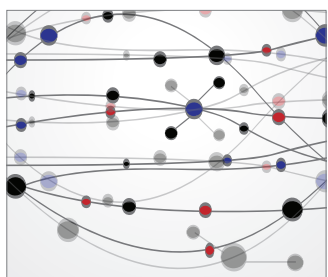

The Scientific World Journal
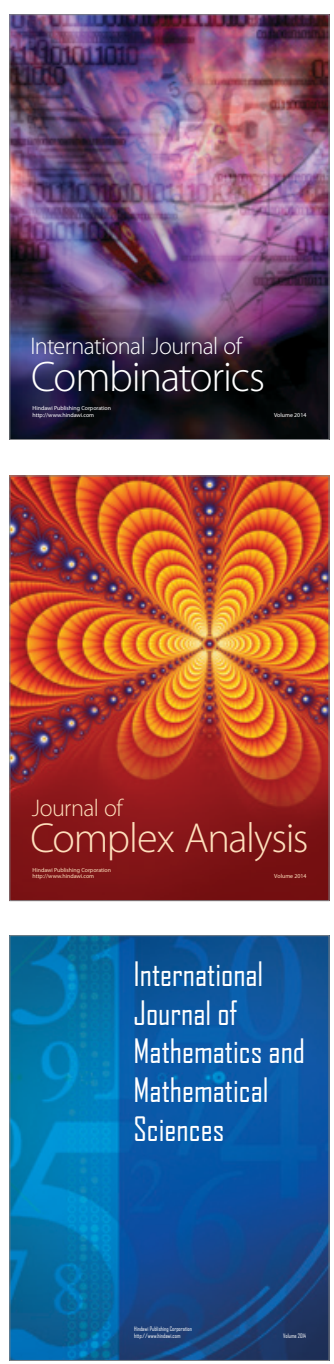
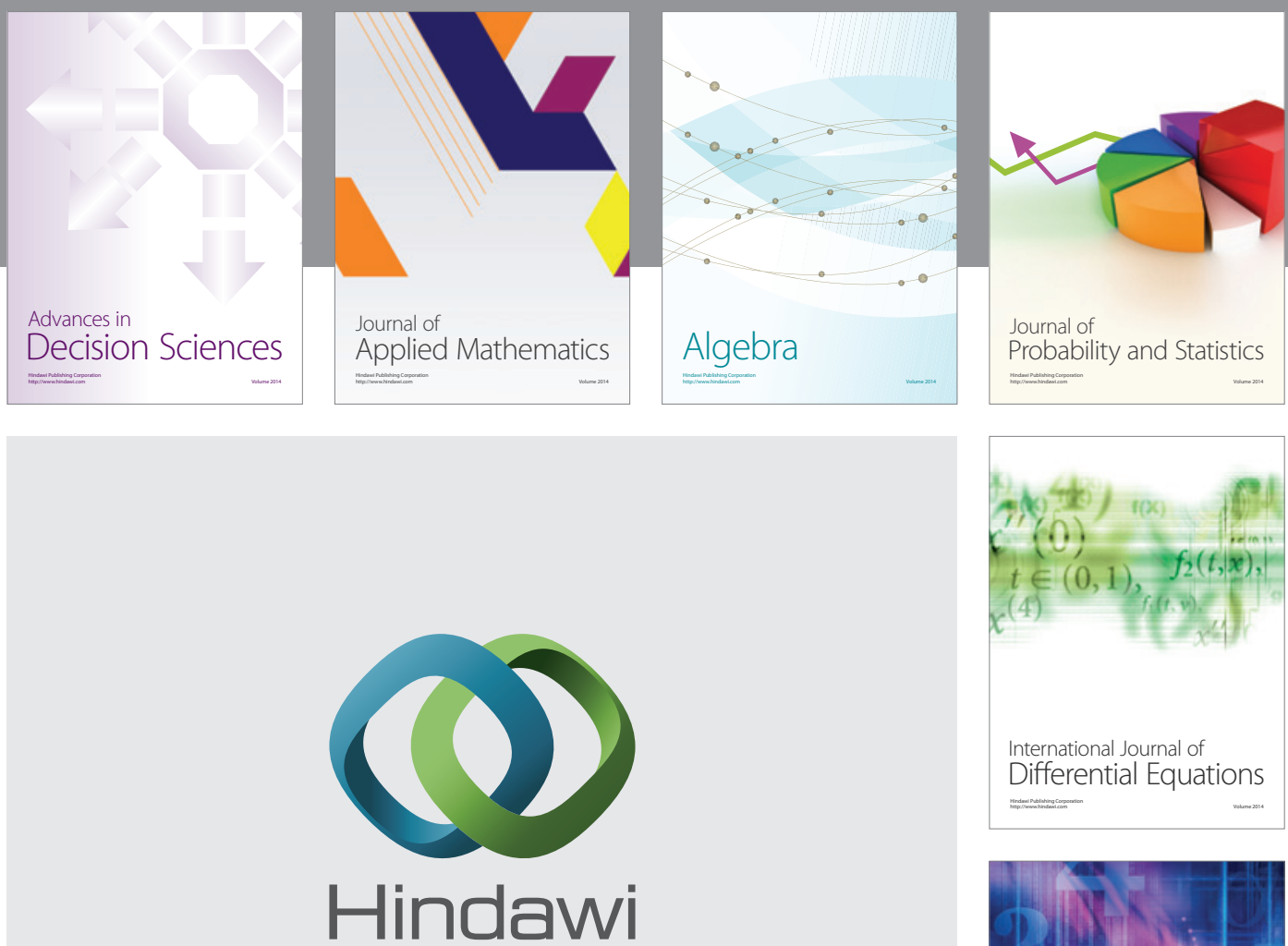

Submit your manuscripts at http://www.hindawi.com
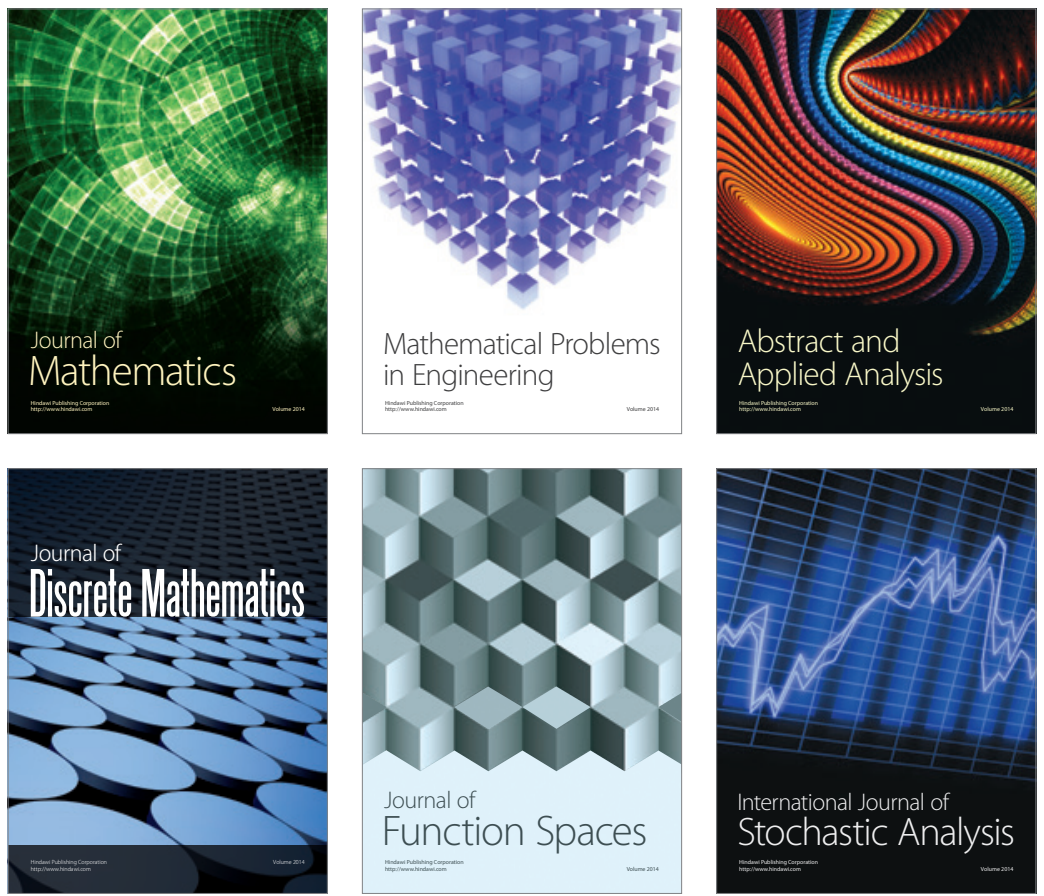

Journal of

Function Spaces

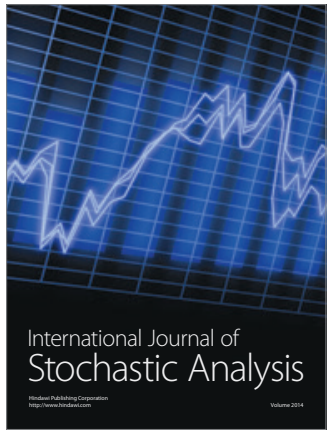

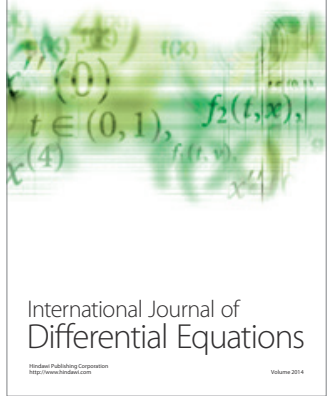
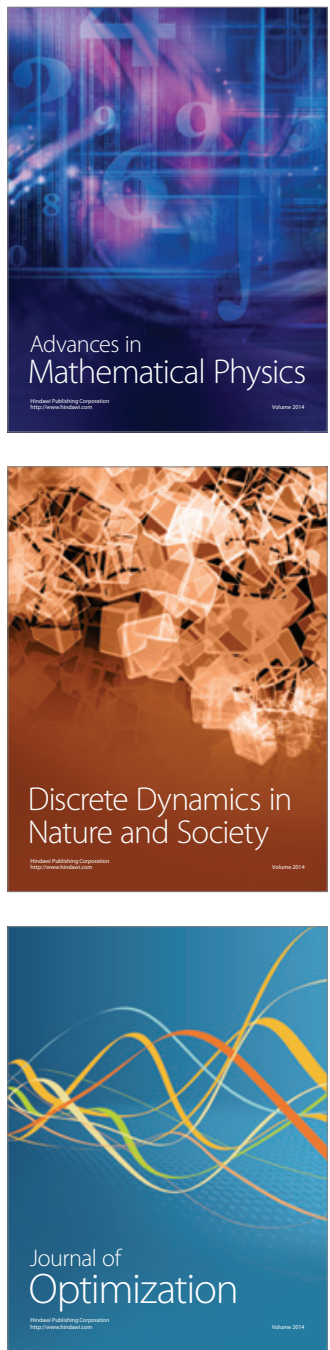